\title{
Una teoría balance de la sabiduría*
}

Robert J. Sternberg

Universidad Estatal de Oklahoma

Recibido: 3 de febrero del 2012 / Aprobado: 15 de marzo del 2012

Aun cuando resulta dificil definir la sabiduría, los psicólogos han desarrollado teorías acerca de este tema; una de ellas es la teoría del balance, que postula que a la hora de tomar decisiones, los individuos sabios se basan en sus valores y conocimientos, que balancean los intereses conflictivos de naturaleza intrapersonal, interpersonal y extrapersonal, en plazos cortos y largos. Este modelo conceptual de la sabiduría está orientado a la acción y toma en cuenta los valores y conocimientos relevantes, sumados a la consideración de los múltiples intereses y a sus posibles consecuencias. Este modelo teórico, además, no solamente puede ser aprendido por los estudiantes, sino también enseñado por los profesores.

sabiduría / teoría balance de la sabiduría

\section{A balance theory of wisdom}

Although it is difficult to define the concept of wisdom, psychologists have developed theories on the concept. One of these is the balance theory of wisdom which states that whence making decisions, wise individuals combine values and knowledge and balance the conflictive nature of intrapersonal, interpersonal and extra-personal interests for short and long terms. This conceptual model of wisdom is action oriented and takes into consideration relevant values and knowledge together with multiple interests and their possible consequences. This model may not only be learned by students but may be taught by instructors..

wisdom / balance theory of wisdom

\footnotetext{
* Traducción del inglés por Ricardo Braun.

Correo electrónico: robert.sternberg@okstate.edu
} 


\section{INTRODUCCIÓN}

Es difícil creer que gerentes de empresas y jefes de Estado que han fracasado en la función de dirigir, hayan podido ser anteriormente profesores universitarios e inclusive rectores. ¿Cómo pueden ser, por un lado, tan inteligentes y al mismo tiempo tan incompetentes en sus posiciones de autoridad? La respuesta es simple: carecen de sabiduría.

\section{¿̇QUÉ ES SABIDURÍA?}

La sabiduría tiene varias definiciones, ninguna de ellas es aceptada enteramente por las personas comunes o los académicos. Se considera a la persona que tiene sabiduría como aquella que tiene habilidad para hacer juicios correctos, para discernir cualidades y relacionar las cosas, un cúmulo de conocimientos filosóficos o científicos, que posee intuición y buen sentido. ¿De qué manera estas definiciones generales se relacionan con modelos teóricos desarrollados acerca de la sabiduría?

Históricamente, el concepto de sabiduría fue el objeto de las investigaciones filosóficas desde los diálogos de $L a$ República de Platón (Robinson, 1990). Recientemente, con la emergencia de la psicología como un campo de estudio separado de la filosofía, el concepto de sabiduría ha sido investigado como un constructo psicológico, y muchos psicólogos han llevado a cabo estudios empíricos acerca de este y sus manifestaciones (Sternberg, 1990; Sternberg
\& Jordan, 2005). La sabiduría ha sido estudiada desde varias perspectivas psicológicas, un resumen de las aproximaciones más relevantes para comprender la sabiduría y una lista de referencias para lectura adicional puede hallarse en Sternberg (2001). Algunos investigadores (Clayton, 1975, 1982; Holliday \& Chandler, 1986; Sternberg, 1990) se han enfocado en teorías implícitas de la sabiduría, esto es, en tratar de entender de qué manera una persona común y corriente percibe y define la sabiduría. Otros han adoptado una perspectiva de desarrollo para investigar cómo la sabiduría se desarrolla o no; tal vez mayor notoriedad cobran los trabajos empíricos que han sido conducidos por Paul Baltes y sus colegas en el Instituto Max Planck (Baltes \& Staudinger, 1993, 2000; Smith \& Baltes, 1990). Otra perspectiva de desarrollo para definir la sabiduría se puede encontrar en el pensamiento operacional posformal, extendiéndose más allá de los estadios piagetanos de la inteligencia (Piaget, 1972).

Varios investigadores y teóricos se han enfocado en la importancia de la integración y el balance en la sabiduría. Labouvie-Vief (1990), por ejemplo, ha enfatizado el balance entre diferentes tipos de pensamiento, sugiriendo que la sabiduría constituye un balance del logos, que son procesos objetivos y lógicos, y mythos, que representa los procesos subjetivos y organísmicos. Kramer (1990) se ha enfocado en el balance entre 
varios auto-sistemas tales como cognitivo, conativo y afectivo, resultando en una personalidad bien balanceada, donde lo consciente e inconsciente interactúan en armonía. Sin embargo, otros insisten en el balance entre diferentes puntos de vista (Kitchener \& Brenner, 1990), o en "un balance entre las valencias opuestas de la emoción intensa y la separación, la acción y la inacción, el conocimiento y las dudas" (Birren \& Fisher, 1990, p. 326).
Este ensayo se enfocará en una teoría de la sabiduría propuesta por Sternberg, que se construye sobre la base de las teorías anteriores pero enfatizando la importancia de la integración y el balance en la sabiduría.

\section{LA TEORÍA BALANCE DE LA SABIDURÍA}

Sternberg define sabiduría como el uso de la inteligencia propia, la creatividad

Figura 1

Dieciséis principios para la enseñanza de la sabiduría (Sternberg, 2001)

Objetivo
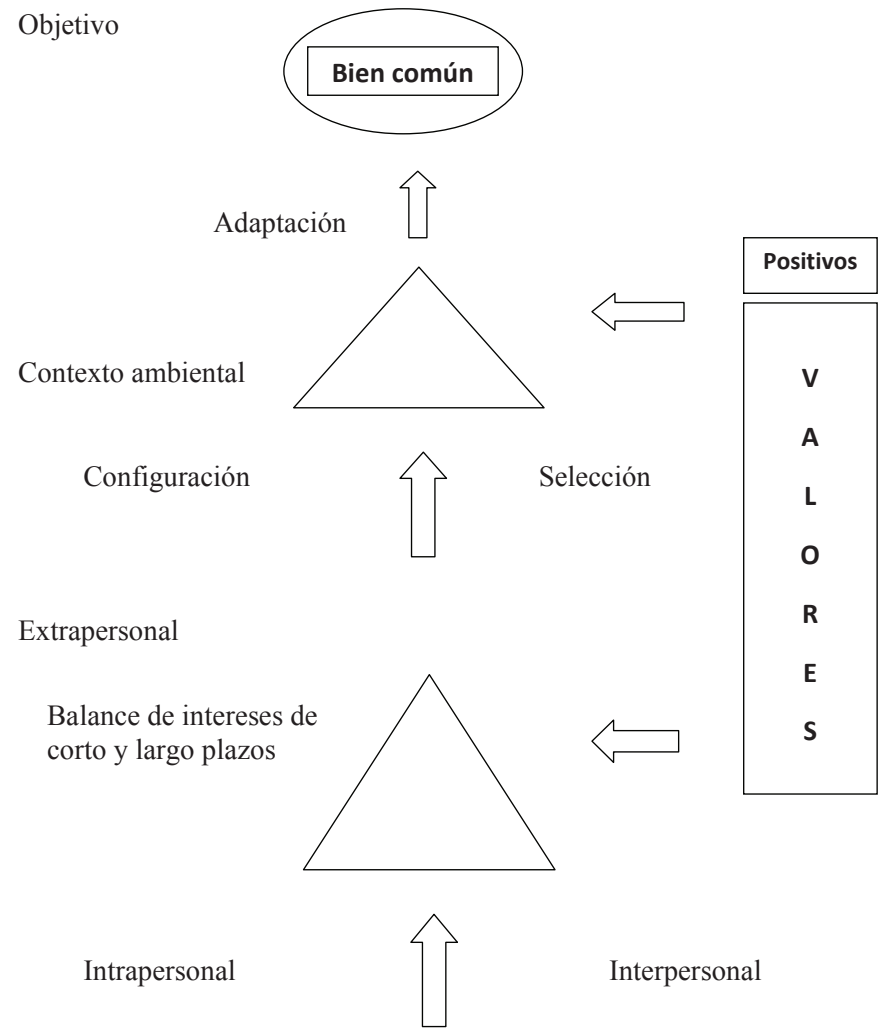

Inteligencia, creatividad y

conocimiento 
y el conocimiento, todo esto mediado por valores positivos hacia el logro de un bien común a través de un balance entre los intereses (a) intrapersonales, (b) interpersonales y (c) extrapersonales; y los plazos (a) cortos y (b) largos para alcanzar un balance entre (a) la adaptación en los entornos existentes, (b) la configuración de los entornos existentes, y (c) la selección de nuevos entornos (Sternberg, 2001, 2003, 2004). La presentación visual de esta concepción de la sabiduría se muestra en la figura 1.

En primer lugar, las decisiones sabias no solo requieren inteligencia y conocimiento explícitos, sino (típicamente) se utilizan conocimientos que son tácitos o implícitos obtenidos también a través de la experiencia. El término conocimiento tácito fue propuesto por primera vez por Polanyi (1966) y describe el conocimiento que es (a) implícito o adquirido sin una ayuda instruccional o aún sin sensación consciente, (b) procedimental, o "saber cómo" en vez de "saber qué", y (c) instrumental para obtener algún objetivo (Sternberg et al., 2000). El conocimiento tácito permite a las personas apreciar ambigüedades de una situación particular que no se podría obtener de ningún conjunto de reglas o bien sea formalizado o aún verbalizado. Es una habilidad para entonarse con las complejidades únicas del rico entorno del individuo y utilizar esa comprensión de estas complejidades para alcanzar los objetivos deseados.
Debo añadir que, aun cuando sea importante, el conocimiento tácito no es un substituto para otros tipos de conocimiento, tales como el conocimiento declarativo o el procedimental explícito. El conocimiento tácito ayuda a dar información para un proceso de decisión sabio en combinación con otros tipos de conocimiento explícito. Por ejemplo, si se les presenta un problema de una disputa limítrofe entre dos países tanto a un diplomático con experiencia y a un destacado estudiante de posgrado en ciencia política con enfoque en política exterior, los dos propondrán soluciones diferentes. Estas diferencias no se fundamentarían en el conocimiento fáctico de los países o en los acontecimientos históricos que condujeron al conflicto, sino más bien en las diferencias de la experiencia con problemas similares y un conocimiento tácito de lo que funciona y lo que no funciona en los casos de arbitraje entre países. Esto no significa que la persona mayor siempre es más sabia. Cuando un profesor de escuela primaria es confrontado con un asunto de manejo en su clase, el joven profesor que tiene tres hijos en casa, puede tener más conocimiento tácito aplicable para resolver el problema que un profesor mayor que no tiene hijos.

En segundo lugar, la definición se sostiene en gran medida en la idea de balance: el balance entre los intereses múltiples, las consecuencias inmediatas y duraderas, y las respuestas ambientales. La mayoría de las teorías psicológi- 
cas de la sabiduría resumidas en la introducción proponen un balance de solo uno de los componentes de la teoría de Sternberg, intereses intrapersonales. En contraste, la teoría de Sternberg aplica la idea de las interacciones dentro y entre las personas y con el ambiente. En otras palabras, el balance necesita existir no solo por los intereses intrapersonales, sino también por los intereses interpersonales y extrapersonales, así como por las respuestas ambientales. ¿Cuáles son estos diferentes intereses y respuestas? Los intereses intrapersonales afectan solo al individuo. Tienen que ver con la noción personal de identidad y pueden incluir tales asuntos como el deseo de autorrealización, popularidad, prestigio, poder, prosperidad o placer. Los intereses interpersonales involucran a otras personas. No solamente se relacionan con la sensación de identidad personal sino también con las relaciones deseables con otras personas. Los intereses extrapersonales son aquellos que afectan organizaciones más amplias, como la comunidad, el país, o el medio ambiente. Además de los múltiples intereses, las consecuencias de cada decisión son evaluadas de manera que se puedan balancear objetivos de corto y largo plazos. Por ejemplo, el diplomático experimentado y el destacado estudiante de posgrado en el caso mencionado pueden ambos saber, a partir de la lectura de libros y de la experiencia, que el país con la fuerza armada más poderosa tiene más posibi- lidades de ganar una disputa fronteriza. Pero si uno considera las consecuencias de largo plazo producidas por el resentimiento del país que fue atacado, o la potencial imagen negativa que puede proyectarse sobre el país atacante, puede ser que la intervención militar no sea la solución más sabia.

Es importante destacar que la teoría de la sabiduría de Sternberg no significa que cada interés, consecuencia, o respuesta tengan el mismo peso. Los "pesos" relativos se determinan por la medida en que una alternativa particular contribuye con el logro de un bien común. Por ejemplo, cuando se trabaja por una solución, los intereses personales (intrapersonales) pueden tener menor peso que los intereses de otros (interpersonales), aun cuando ambos forman parte de la decisión final. Como ilustración, cuando el profesor de clase descrito anteriormente trata de decidir si debe enviar o no al estudiante que perturba la clase a la dirección del colegio, puede considerar su propio interés en que otra persona se encargue del problema como también los intereses de otras personas de la escuela (por ejemplo, la dirección). También puede pensar en las consecuencias de corto plazo (por ejemplo, la expulsión inmediata del elemento perturbador de la clase) y las consecuencias de largo plazo (por ejemplo, enviar el mensaje a sus estudiantes de que si uno quiere dejar la clase lo único que tiene que hacer es provocar ruido y serán enviados a la dirección). Una per- 
sona sabia tomará en cuenta todas estas consideraciones. Más aún, él o ella pueden tratar de balancear los múltiples intereses y consecuencias de tal modo que pueda maximizar el bien común. Por ejemplo, el profesor puede separar al estudiante problema de la actividad grupal y asignarle la tarea de completar un conjunto de ejercicios en el fondo de la clase, pesando de esta manera en menor grado el interés personal y la inmediata satisfacción, sin ignorarlos totalmente.

Lo que se hace evidente, aun con ejemplos triviales como los mencionados, es que la elección del balance correcto depende del sistema individual de valores. En efecto, los valores se encuentran en el núcleo del proceso de decisión, y no solo en la teoría balance que se describe aquí. De acuerdo con Csikszentmihalyi y Rathunde (1990, p. 32), "la sabiduría se convierte en la mejor guía de lo que es el súmmum bonum o el "bien supremo". Pascual-Leone (1990) también considera "los sentimientos y evaluaciones éticas (juicios correctosincorrectos o buenos-malos) de las motivaciones y de los actos posibles (por ejemplo, moralidad)" como un importante componente de la sabiduría (véase Sternberg \& Stemler, 2004, p. 267). En la teoría de Sternberg, los valores no solamente establecen lo que constituye el bien común, sino que también influyen los pesos relativos de los diferentes intereses, las consecuencias conflictivas y las respuestas alternativas al entorno.
El lugar central de los valores positivos de la teoría de Sternberg pone en discusión la pregunta acerca de quién determina cuáles son los valores positivos "correctos". Sabemos que los valores de las personas difieren en distintas culturas y en diversos periodos de la historia. De hecho, nuestro propio sistema democrático demanda que respetemos las diferencias de los demás cuando decidimos lo que es correcto e incorrecto. El peligro en este caso es sucumbir a un relativismo moral y que se sostenga que cualquier sistema valorativo es igualmente justificable y que no hay un principio que permita distinguir lo bueno de lo malo. Argumentaría que aun cuando podría haber más de un sistema de valores aceptable, existen otros que debieran ser rechazadas bajo la fundamentación de que dejan de reconocer las libertades humanas fundamentales. En otras palabras, el respeto por las diferencias de otras personas no puede extenderse más allá del respeto de los valores humanos nucleares, tales como la vida y la imparcialidad. Además, no pretendo definir una lista exhaustiva de los valores que debieran ser respetados y reconocidos por cada cultura, pero sí me reservo el derecho de destacar explícitamente algunos valores, por ejemplo, el respeto por la vida humana, cuyo incumplimiento es inaceptable y poco sabio.

Cuando se enfrenta un problema, los individuos sabios se basan en sus valores y conocimiento que ayude a 
encontrar una solución que balancee los intereses conflictivos de naturaleza intrapersonal, interpersonal y extrapersonal en plazos cortos y largos. Este modelo conceptual de la sabiduría, sin embargo, no es meramente un ejercicio intelectual esotérico. Más bien, es un modelo orientado a la acción. La aplicación de los valores y conocimiento relevantes, sumados a la consideración de los múltiples intereses y consecuencias, debe conducir a la elección de una conducta determinada. Nuevamente, en este caso, la teoría se nutre de la idea del balance: un balance entre la adaptación, la forma y la selección de los entornos. La adaptación supone el cambio de uno mismo para adaptarse al entorno ya existente. Por ejemplo, el profesor en el caso mencionado puede hacer arreglos en su salón de clases para que haya un lugar silencioso para el trabajo individual para ser asignado a aquellos estudiantes que provocan desorden. $\mathrm{O}$ puede solicitar ayuda para que alguien pueda trabajar individualmente con aquellos estudiantes que no funcionan adecuadamente en trabajos grupales. La forma es el comportamiento opuesto, supone modificar aquellas partes del entorno para que se adecúen a los intereses propios. Por ejemplo, el profesor puede trabajar con otros en la escuela para lograr asistencia adicional con el fin de enfrentar asuntos de manejo de clase. Puede pedir entrenamiento profesional en el asunto, o que una sección de la biblioteca pueda ser reservada para la tutoría individualizada de los estudiantes problemáticos, o que los padres de familia asistan más frecuentemente para las conferencias padres-profesores y así ayudar a monitorear el comportamiento de sus hijos en la escuela. Finalmente, si las personas no pueden adaptarse al ambiente existente o no pueden cambiar el ambiente para que esté de acuerdo con sus necesidades, ellos pueden seleccionar un nuevo ambiente. El profesor puede escoger cambiarlos a una escuela diferente donde haya menores problemas de manejo de clase. Una solución específica puede también combinar las respuestas ambientales, tales como la adaptación y la forma, como cuando conjuntamente uno hace cambios personales y de ambiente.

Parecería que el acto de balance entre los intereses, consecuencias y respuestas al ambiente se ha convertido en algo abrumadoramente complejo y que no nos ayuda a comprender los procesos psicológicos y resultados involucrados en la toma de decisión sabia. Algunos autores (Paris, 2001) han criticado el modelo porque convierte el concepto de sabiduría en algo más oscuro y difícil de comprender, en lugar de lo contrario (para una discusión véase Reznitskaya \& Sternberg, 2004). La presencia del balance, los valores y el conocimiento en una conceptualización del pensamiento sabio no es exclusiva de la teoría de Sternberg. Lo que es exclusivo es la proposición que incluye todos los factores relevantes en el proceso de los 
juicios sabios, así como la explicación de la relación entre esos factores. El valor de la teoría balance de la sabiduría proviene de su inteligibilidad en la descripción de uno de los actos más sofisticados de la mente humana. No creo que sea posible diseccionar el pensamiento sabio en fracciones convenientemente manejables. El modelo teorético es complejo porque el comportamiento real en ambientes reales es complejo.

Sin embargo, se debiera recordar que cada decisión tiene lugar en un contexto particular donde, realistamente, hay un número finito de intereses en competencia, posibles consecuencias y respuestas ambientales disponibles. La teoría balance de la sabiduría permite que uno evalúe una decisión dentro de un contexto específico considerando todos los factores enumerados y descritos en el modelo conceptual del proceso de toma de decisión. Aun cuando la teoría balance de la sabiduría no puede determinar la respuesta sabia a cualquier problema, puede ayudar a evaluar cuan bien una solución particular cumple con las especificaciones de la teoría en un contexto dado.

Se podría argumentar correctamente que la formulación de una solución sabia a un problema no conlleva necesariamente a actuar de acuerdo a ella. Podemos pensar en ejemplos personales en los que una clara conciencia de las potenciales consecuencias dañinas no evitó que realizáramos una acción que era atractiva de alguna forma en el momento. Esta discrepancia entre la conciencia y el comportamiento puede ser tomada en cuenta con la inclusión de los intereses intrapersonales en el modelo. Una acción sabia, como una solución sabia, requerirá un balance entre los intereses intrapersonales, que puede incluir la resolución de la tensión entre varios llamados afectivos y cognitivos. Perkins (2002) describe varias estrategias para confrontar comportamientos que uno considera poco sabios, pero que, sin embargo, encuentras muy irresistible abandonarlos. Por ejemplo, comportamientos como la impulsividad, la procrastinación, la complacencia o la indecisión pueden ser disminuidos y aun eliminados con el uso de condicionamiento deliberado o técnicas de autoadministración.

Considerando la complejidad del marco teórico y los posibles obstáculos para encontrar soluciones sabias y actuar sabiamente, ipueden y deben las escuelas enseñar de una forma que se incremente el pensamiento sabio en sus estudiantes?

\section{LAS SEIS FALACIAS DE LA TONTERÍA}

La falta de sabiduría es tontería. El comportamiento tonto, sugiero, se debe en gran medida, aunque no exclusivamente, a seis falacias del pensamiento. Estas falacias se parecen a las que asociamos con el pensamiento adolescente, porque son del tipo de pensamiento que usualmente se observa en los adolescentes. 
- La falacia del optimismo irreal.Esta falacia ocurre cuando se cree que uno es tan inteligente o poderoso que no tiene sentido preocuparse por los resultados, y especialmente los de largo plazo, de lo que uno ha hecho puesto que al final todo saldrá bien; no hay nada de qué preocuparse, dado el cerebro de uno o el poder. Si uno simplemente actúa, el resultado será correcto. Bill Clinton tendía a repetir su comportamiento sexual que, primero como gobernador y luego como presidente, llevaría a un mal final. No parecía preocuparse por ello.

- La falacia del egocentrismo. Esta falacia ocurre cuando uno piensa que sus propios intereses son los únicos importantes. Se empieza ignorando las propias responsabilidades hacia otras personas o instituciones. Algunas veces, las personas en posiciones de responsabilidad pueden empezar con buenas intenciones, pero se corrompen con el poder que ejercen y su ausencia de rendición de cuentas hacia otros. Un primer ministro, por ejemplo, puede usar su cargo en parte o incluso principalmente para escapar de un proceso judicial, como aparentemente ha ocurrido en algunos países europeos y sudamericanos en los últimos años.

- La falacia omnisciente.- Esta falacia resulta de tener a su disposición esencialmente cualquier conocimiento que uno quiere, que es, de hecho, adquirible. Con una llamada telefónica, un líder poderoso puede tener todo tipo de conocimiento accesible. Al mismo tiempo, las personas aprecian a los líderes poderosos que son extremadamente expertos o cercanos a la omnisciencia. El líder poderoso comenzará a creer que él o ella son realmente omniscientes. Y también sus subordinados.

- La falacia omnipotente.- Esta falacia resulta del extremo poder que se ejerce, o cree que se ejerce. El resultado es el abuso de poder. Algunas veces, los líderes crean enemigos internos o externos para demandar más poder para lidiar con los supuestos enemigos. En Estados Unidos, en el 2008, el gobierno central se arrogó más poder del que había tenido cualquier gobierno en la historia reciente sobre la base de las supuestas amenazas terroristas. En Zimbabue, Robert Mugabe enfrentó un grupo contra otro, con el objetivo similar de expandir sustancialmente y mantener su propio poder.

- La falacia de la invulnerabilidad. Esta falacia se deriva de la presencia de la ilusión de la protección completa, tal como la que proporciona una administración numerosa. Las personas, y especialmente los líderes, pueden aparentar tener muchos amigos prestos a protegerlos en el momento que se les requiera. Los líderes pueden protegerse de aquellos individuos que sean menos serviles. 
- La falacia de la desconexión ética. Esta falacia ocurre cuando se empieza a creer que la ética es importante para cualquier persona menos para uno mismo. Muchos líderes de países y corporaciones se creen exceptuados de los estándares éticos que sí consideran válidos para los demás.

\section{¿̇Podemos enseñar PARA LA SABIDURÍA?}

La educación occidental en los últimos dos siglos se ha enfocado típicamente a impartir conocimientos así como al desarrollo de habilidades cognitivas en los estudiantes. Las escuelas promueven entre sus estudiantes la inteligencia, pero no necesariamente la sabiduría. Estos estudiantes pueden tener magníficos resultados en la escuela, sin embargo hacen juicios pobres en sus propias vidas y en las vidas de otras personas. Un objetivo importante de los educadores, creo, es ayudarles a llevar vidas felices, satisfactorias y productivas. Un creciente número tanto de investigadores como de ejecutores de políticas comparten la creencia de que las escuelas deben promover el desarrollo cognitivo y moral de sus estudiantes (Reznitsjkaya \& Sternberg, 2004). Llevar a cabo una vida exitosa inevitablemente conlleva la habilidad para resolver problemas difíciles e inciertos de la vida diaria. Los problemas a los que se exponen las personas varían según su entorno y las responsabilidades que acarrean, pero cualquier persona en algún momento se expondrá a situaciones en las que tendrá que depender de la sabiduría para tomar la decisión correcta. Por eso, creemos que la escuela debiera ayudar a promover las habilidades del pensamiento sabio en sus estudiantes.

A primera vista, el modelo de sabiduría de Sternberg podría parecer inadecuado para la educación formal. El fuerte énfasis que se coloca en la teoría balance sobre los valores, el conocimiento tácito y las interacciones específicas del contexto entre la persona y su entorno podría parecer que impide su aplicación en el ambiente de clase. ¿Cómo podrían los profesores ayudar a sus estudiantes a desarrollar todas las intuiciones explícitas e implícitas que se requieren para la aparición de la sabiduría?

La meta de la enseñanza para la sabiduría puede ser alcanzada proporcionando a los estudiantes contextos educacionales en los que puedan formular su propia comprensión de lo que constituye el pensamiento sabio. En otras palabras, la enseñanza para la sabiduría no se obtiene a través de un método didáctico en el que se "imparte" información acerca de la sabiduría, y subsecuentemente evaluando a los estudiantes con preguntas de opción múltiple. En vez de eso, los estudiantes necesitan experimentar activamente varios procesos cognitivos y afectivos que subyacen a las decisiones sabias. En otras palabras, los estudiantes pueden proporcionar un andamiaje para el desarrollo de la sabiduría y los estudios de casos pueden ayudarlos a desarrollar la sabiduría, 
pero el profesor no puede enseñar caminos particulares de acción, o dar a los estudiantes una lista de hacer o no hacer, al margen de las circunstancias.

¿Cuáles son los procesos subyacentes al pensamiento sabio que los estudiantes deben adquirir, y cómo pueden ser introducidos en la clase? Sternberg
(2001) reseña dieciséis principios pedagógicos y seis procedimientos derivados de la teoría de la sabiduría, descrito en las tablas 1 y 2 . La idea fundamental detrás de estas guías educacionales es que el docente enseña a los estudiantes no lo que deben pensar, sino más bien, cómo deben pensar.

Tabla 1

Dieciséis principios para la enseñanza de la sabiduría (Sternberg, 2001)

\begin{abstract}
Principios 1-8
Principio 1

Explorar con los estudiantes la noción de que las habilidades convencionales no son suficientes para una vida satisfactoria. Muchas personas se encuentran atrapadas en sus vidas $\mathrm{y}$, a pesar de sentirse convencionalmente satisfechas, perciben que sus vidas carecen de realización. La realización no es una alternativa al éxito, sino más bien un aspecto de él, que para la mayoría de personas, va más allá del dinero, de la promoción profesional, de una vivienda grande, y así sucesivamente.
\end{abstract}

\section{Principio 2}

Demostrar cómo la sabiduría es de importancia crítica para una vida satisfactoria. En el largo plazo, las decisiones sabias benefician a las personas en formas que las decisiones tontas nunca lo podrán hacer.

\section{Principio 3}

Enseñar a los estudiantes la utilidad de la interdependencia; una marea alta puede elevar todos los barcos, una marea baja puede hundirlos.

\section{Principios 9-16}

\section{Principio 9}

Ayudar a los estudiantes a aprender los roles de adaptación, configuración y selección, y cómo balancearlos. Los juicios sabios dependen en parte de la selección entre respuestas ambientales. 


\section{Principio 4}

Ser modelo de rol de la sabiduría puesto que lo que uno hace es más importante de lo que se dice. La sabiduría es dependiente de la acción y las acciones sabias requieren ser demostradas.

\section{Principio 5}

Hacer que los estudiantes lean acerca de juicios sabios y toma de decisiones, de modo que puedan comprender que existen tales modos de juicio y toma de decisiones.

\section{Principio 12}

Mostrar a los estudiantes la importancia del pensamiento dialógico, con el que pueden comprender los intereses e ideas desde diferentes puntos de vista.

\section{Principio 6}

Ayudar a los estudiantes a aprender a reconocer sus propios intereses, aquellos de otras personas, y aquellos de las instituciones.

\section{Principio 13}

Enseñar a los estudiantes a buscar y tratar de alcanzar el bien común, un bien en el que todos ganan y no solo aquellos con los que uno se identifica.

\section{Principio 7}

Ayudar a los estudiantes a balancear sus propios intereses con aquellos de otras personas y aquellos de las instituciones.

\section{Principio 14}

Estimular y premiar la sabiduría.

\section{Principio 8}

Enseñar a los estudiantes que los medios por los que se obtienen los fines son importantes, y no solo los fines.

\section{Principio 15}

Enseñar a los estudiantes a monitorear los acontecimientos de la vida y sus propios procesos de pensamiento acerca de esos eventos. Una forma de aprender a reconocer los intereses de otros es comenzando por identificar los propios.
Revisemos los seis procedimientos para enseñar la sabiduría presentados en la tabla 2 con más detalle.

- Procedimiento 1.- Fomente entre sus estudiantes la lectura de obras clásicas de la literatura y la filosofía

\section{Principio 16}

Ayudar a los estudiantes a comprender la importancia de inocularse a sí mismo contra las presiones del interés individual y el interés de grupos pequeños. (sea occidental o de otra tradición) para aprender y reflexionar sobre la sabiduría. El apuro por desechar las obras clásicas por obras modernas solo tiene sentido si la sabiduría que estas obras modernas pueden 
Tabla 2

Seis procedimientos para la enseñanza de la sabiduría (Sternberg, 2001)

\begin{tabular}{|c|c|}
\hline & Procedimientos \\
\hline 1 & $\begin{array}{l}\text { Alentar a los estudiantes a leer obras clásicas de la literatura y la filosofía para que } \\
\text { aprendan y reflexionen sobre la sabiduría de los sabios. }\end{array}$ \\
\hline 2 & $\begin{array}{l}\text { Estimular a los estudiantes en las discusiones en clase, proyectos y ensayos que } \\
\text { promuevan la discusión de las lecciones que han aprendido de esas obras y cómo } \\
\text { se pueden aplicar en sus propias vidas y las vidas de otros. Un énfasis particular } \\
\text { debe ser puesto en el pensamiento tanto dialógico (principio 12) y dialéctico (prin- } \\
\text { cipio 11). }\end{array}$ \\
\hline 3 & $\begin{array}{l}\text { Alentar a los estudiantes a estudiar no solamente la "verdad" sino también los va- } \\
\text { lores, cómo se desarrollan durante el proceso de pensamiento reflexivo. }\end{array}$ \\
\hline 4 & $\begin{array}{l}\text { Poner un mayor énfasis en el pensamiento crítico, creativo y práctico, con el objetivo } \\
\text { de que los buenos fines beneficien al bien común. }\end{array}$ \\
\hline 5 & $\begin{array}{l}\text { Alentar a los estudiantes a pensar en que casi cualquier tema puede ser usado para } \\
\text { fines buenos o malos, y acerca de lo importante que es el objetivo final. }\end{array}$ \\
\hline 6 & $\begin{array}{l}\text { ¡Recordar que el docente es un modelo de rol! Para modelo de rol de la sabiduría, } \\
\text { el docente debe adoptar un enfoque socrático en la enseñanza, e invitar a los } \\
\text { estudiantes a jugar un papel más activo en la construcción del conocimiento, desde } \\
\text { su punto de vista y el de otros. }\end{array}$ \\
\hline
\end{tabular}

impartir iguala o supera aquella de las obras clásicas. Cuando sea posible, fomente en sus estudiantes que participen en el pensamiento reflexivo, para reflejar en su propio funcionamiento el incremento de su metacognición (Flavell, 1987), esto es, su conciencia de sus cogniciones, emociones y creencias. El proceso de tomar una decisión sabia es estratégica y orientada hacia la meta, y por lo tanto, requiere un monitoreo constante de las estrategias escogidas, así como la habilidad para mo- dificar las estrategias menos exitosas y estar preparados para demandas situacionales. Los profesores pueden ayudar a sus estudiantes a practicar el pensamiento reflexivo diseñando actividades instruccionales que les permitan a ellos explorar y modelar sus propios valores. Además, se les puede instruir explícitamente en estrategias metacognitivas, tales como el autocuestionario o el uso de listas de chequeo de automonitoreo.

- Procedimiento 2.- Promueva la participación de los estudiantes en las 
discusiones de clase, en proyectos $\mathrm{y}$ ensayos que fomenten la discusión de las lecciones que recién han aprendido de las obras literarias y filosóficas leídas, y cómo esas lecciones pueden ser aplicadas en sus propias vidas y en las de los demás. Un currículo de historia, por ejemplo, debe privilegiar las relaciones entre la historia y las experiencias cotidianas personales relevantes.

- Los docentes deben participar en el pensamiento dialógico y dialéctico, además del pensamiento reflexivo anteriormente descrito. ¿Qué es el pensamiento dialógico (principio 12) (véase la tabla 1)? Cuando uno se enfrenta a un problema complejo que incluye varios puntos de vista, usualmente es necesario tomar en consideración diferentes marcos de referencia y varias perspectivas para encontrar la mejor solución posible. Lo que a primera vista puede aparecer como la respuesta correcta puede resultar una opción incorrecta, si se toma el largo plazo, o cuando los intereses de la comunidad tomada como un todo se toman en cuenta. En el pensamiento dialógico, uno utiliza múltiples marcos de referencia para generar y deliberar acerca de diferentes perspectivas del problema en cuestión. Las soluciones óptimas provienen de sopesar las alternativas, en lugar de seguir un solo curso de actividades prescritas. En la clase, los profesores pueden alimen- tar la habilidad de los estudiantes para pensar dialógicamente a través de actividades en las que varios puntos de vistas se presentan y discuten. Algunos estudios empíricos que han investigado la efectividad de las discusiones de los estudiantes para el desarrollo del pensamiento dialógico, demuestran mejora en la habilidad de los estudiantes para resolver problemas mal estructurados después de su participación en las discusiones (Kuhn, Shaw, \& Felton, 1997; Reznitskaya et al., 2001).

- ¿En qué consiste el pensamiento dialéctico (principio 11)? Mientras que el pensamiento dialógico involucra la consideración y peso de los múltiples puntos de vista, el pensamiento dialéctico enfatiza la consideración e integración de dos perspectivas opuestas. La primera perspectiva considerada es la tesis. Por ejemplo, uno puede ser un pacifista radical y opuesto a la presencia militar, sean cuales fueren las circunstancias. Una segunda perspectiva, la antitesis (una negación de la proposición original) es ahora considerada. Por ejemplo, uno puede argumentar que las personas solo pueden vivir con libertad y en paz si sus fronteras están protegidas por fuerzas armadas. Finalmente, se desarrolla una sintesis o reconciliación de las dos proposiciones aparentemente opuestas. Por ejemplo, cuando uno puede decidir que las fronteras en disputa 
deben ser protegidas por una tercera parte, como una fuerza armada internacional, en vez de que los países en conflicto midan su fuerza militar entre ellos. El proceso no se detiene cuando las dos posiciones opuestas se reconcilian; al contrario, cada síntesis se convierte en una nueva tesis, que puede ser integrada en una nueva ronda de pensamiento dialéctico. En el aula, el pensamiento dialéctico puede ser promovido a través de oportunidades para estudiar diferentes fuentes, permitiendo de esta manera que los estudiantes construyan su propio conocimiento, o a través de trabajos escritos que explícitamente requiera la formulación de una tesis, una antítesis y una síntesis. Los estudios empíricos han investigado el impacto que produce el desarrollo de este flujo y el concepto dinámico del conocimiento, donde la fuente del conocimiento no es la "autoridad" (el docente o el libro), sino más bien, el estudiante. Tales concepciones de conocimiento demuestran que existe una relación de compromiso activo en el aprendizaje (por ejemplo, McDevitt, 1990), persistencia en la ejecución de una tarea (por ejemplo, Dweck \& Leggett, 1988), y una comprensión más profunda e integración del material enseñado (por ejemplo, Qian \& Alvermann, 2000; Songer \& Linn, 1991).

- Procedimiento 3.- Alentar a los estudiantes a estudiar no solamente la "verdad", sino valores, tal como se van desarrollando durante el pensamiento reflexivo.

- Procedimiento 4.- Poner cada vez mayor énfasis en el pensamiento crítico, creativo y práctico para promover los buenos fines que benefician el bien común. En una clase típica, los profesores deben estimular las habilidades del pensamiento crítico entre sus estudiantes. Algunos docentes también buscan el desarrollo de habilidades de pensamiento creativo y práctico (Sternberg \& Grigorenko, 2007) comprometiendo a sus estudiantes en actividades que los conducen a ir más allá del contenido que han estudiado (pensamiento creativo) para aplicar este conocimiento en su entorno (pensamiento práctico). Para mejorar el pensamiento sabio, sin embargo, los estudiantes deben ser estimulados para tomar en cuenta el resultado de su pensamiento, y tener en mente que la mejor solución no es siempre lo que beneficia solo al individuo sino que es uno que ayuda a otros también. El bien común debiera ser el principio que guía cuando se escoge entre diferentes posibles soluciones.

- Procedimiento 5.- Animar a los estudiantes a pensar que casi cualquier tema que estudian puede ser usado para fines mejores o peores, y acerca de cuan importante es el objetivo final. Como se describió en el pro- 
cedimiento 4 , se debe animar a los estudiantes a buscar diferentes soluciones y escoger aquella que beneficia el bien común antes que el individual. También se debería provocar la conciencia de que, tal como hay diferentes soluciones que benefician a diferentes personas, un concepto dado o una posición de conocimiento puede ser para una finalidad buena o mala. Un ejemplo estereotípico es que el conocimiento de la física nuclear puede ser usado para construir bombas o para desarrollar fuentes de energía. El fin que uno escoge para aplicar el conocimiento que se tiene es algo que importa mucho.

- Procedimiento 6.- ¡Debe recordarse que el docente es un modelo de rol! Para el modelo de rol de sabiduría, el profesor debe adoptar la perspectiva socrática en la enseñanza, e invitar a los estudiantes a jugar un rol más activo en la construcción del conocimiento, desde su propio punto de vista y desde el de otros. El pensamiento sabio no es un conjunto de reglas o decisiones que el docente puede esquematizar para que los estudiantes lo copien, es un tipo de pensamiento que los estudiantes deben adoptar y dominar. La forma más efectiva de promover las habilidades de pensamiento sabio no es mediante la repetición de memoria sino a través de la participación estudiantil y el rol de modelo por parte del docente. Por ejemplo, un profesor pue- de capitalizar a partir de un acontecimiento negativo -dos estudiantes que han iniciado una pelea- como una manera de demostrar cómo uno puede abordar una situación similar en una forma más constructiva. El profesor puede modelar el pensamiento sabio diciendo: "Cuando me encuentro en una situación como esta, trato de ver la disputa desde una perspectiva de la otra persona y pienso si mi propio comportamiento contribuye a la situación. ¿Existe alguna forma diferente en la que pude haber actuado para prevenir esta confrontación? ¿Hay una solución para nuestras discrepancias que sea aceptable para las dos partes?'. Asimismo, los docentes no deben perder la oportunidad para reconocer y felicitar los buenos juicios elaborados por los estudiantes, tal como cuando se muestra aprecio por los demás y sus ideas, o cuando ofrecen una solución que beneficia a la clase como un todo en vez del beneficio al individuo. El docente más efectivo es aquel que puede crear una comunidad de clase en la que se practica la sabiduría, en vez de simplemente predicarla. Los estudiantes necesitan experimentar que la sabiduría puede ser alcanzada en la vida real y no en el papel, y que puede conducir a una existencia más armoniosa.

En la enseñanza de la ciencia, el pensamiento dialéctico puede ser aplicado para ilustrar a los estudiantes so- 
bre la noción de que los hechos científicos no son eternos ni inmutables, sino más bien un estado de cosas tal como lo percibimos en un periodo específico de tiempo. En efecto, usualmente se presenta a la ciencia como si representara el fin de un proceso de evolución del pensamiento y no como uno de varios puntos intermedios (Sternberg, 1998). Así presentada, difícilmente los estudiantes podrán darse cuenta de que los paradigmas actuales, y en consecuencia las teorías y descubrimientos que emanan de ellos, eventualmente serán superados, como los paradigmas, teorías $\mathrm{y}$ descubrimientos del pasado fueron reemplazados por aquellos de la actualidad. Más aún, los estudiantes deben aprender que, contrariamente a la forma como se presentan en los libros de texto, el "método científico" clásico es un ideal y no una realidad, y que los científicos son susceptibles de ser tan pasajeros como cualquier otra persona. ¿Cuántos científicos de su época consideraron como evidencia científica los datos presentados por Galileo Galilei para demostrar que la Tierra circulaba alrededor del Sol y no viceversa?

Las habilidades del pensamiento sabio pueden ser aplicadas también en las clases de literatura. La literatura se enseña frecuentemente en términos de los estándares y contexto de la escena estadounidense contemporánea. Los personajes son juzgados en relación con nuestros estándares contemporáneos en vez de los estándares del tiempo y lu- gar en que sucedieron. Imaginemos que los estudiantes abordaran usualmente el estudio de las obras literarias en un entorno dialógico, estudiando la literatura en el contexto de la historia. La censura y la abolición de los libros frecuentemente reflejan la aplicación de ciertos estándares contemporáneos a la literatura, de los que el autor nunca podría haber estado consciente.

La clase de lengua extranjera también es un ambiente en el que se pueden mejorar las habilidades de pensamiento sabio. Las lenguas extranjeras deben ser enseñadas en el contexto cultural en el que se hallan, requiriendo de los estudiantes la necesidad de compenetrarse en el pensamiento reflexivo y dialógico para captar realmente la cultura foránea y colocarse ellos mismos y sus experiencias en relación con esa cultura. Suele ser más común en Europa hablar una o varias lenguas aparte de la lengua materna. Quizás es por ello que los estudiantes de Estados Unidos tienen mayor dificultad de aprender lenguas extranjeras que sus pares de Europa, no porque carezcan de habilidad sino porque carecen de la motivación y exposición. Un estudiante estadounidense probablemente vería la necesidad de aprender una lengua extranjera si cada uno de los cincuenta estados hablara una lengua distinta, como sucede en los países de la Unión Europea. Haríamos un gran favor a nuestros estudiantes enseñándoles a comprender otras culturas en lugar de simplemente esperar 
que las personas de otras culturas los entiendan. Aprender el lenguaje de una cultura es la llave para comprenderla, y lenguaje y cultura no se pueden enseñar de forma separada, o considerando la cultura como un apéndice de la lengua en vez de ver el lenguaje en el contexto en el que se encuentra profundamente enraizado.

\section{¿̇Deberíamos enseñar para la SABIDURÍA?}

Enseñar para la sabiduría no solamente engrandece las habilidades de pensamiento de los estudiantes -su habilidad para razonar reflexiva, dialógica y dialécticamente- sino también ayuda a los educadores a desarrollar las unidades del currículo en forma más integrada. Las unidades integradas son beneficiosas porque ayudan a los estudiantes a ver la imagen más grande y comprender cómo la literatura se relaciona con la historia, cómo la ciencia, los descubrimientos y los hechos científicos están insertados en un tiempo y un espacio específicos, cómo la ciencia social y las políticas sociales se relacionan con la historia y la geografía, cómo la economía está influenciada por las creencias filosóficas y políticas así como por el clima y la geografía, o cómo la lengua extranjera es inseparable de la cultura. Aun entre las mismas disciplinas se requiere más integración para que los estudiantes adquieran una comprensión completa y compleja de un tema. Los diferentes enfoques de la psicolo- gía, por ejemplo, se enseñan en forma de competencia cuando, de hecho, son totalmente compatibles. Por eso, las psicologías biológica, cognitiva, del desarrollo, social y clínica proporcionan puntos de vista complementarios de los seres humanos. No compiten una con otra para convertirse en el "punto de vista correcto". El estudio del cerebro es importante, por ejemplo, pero mucho de la comprensión acerca del aprendizaje y la memoria que puede ser aplicado a la enseñanza ha provenido de los enfoques conductistas y cognitivos, y no del enfoque biológico.

Para resumir y concluir este ensayo, afirmo cuatro razones por las cuales las escuelas debieran incluir educación en habilidades de pensamiento sabio en su currículo:

a) El conocimiento es insuficiente para la sabiduría y ciertamente no garantiza la satisfacción o la felicidad. La sabiduría parece ser un mejor vehículo para el logro de esos fines.

b) La sabiduría proporciona una forma consciente y considerada para incorporar valores reflexivos y deliberados en juicios importantes. Uno no puede ser sabio y a la vez impulsivo $o$ inconsciente en los juicios personales.

c) La sabiduría representa una vía para crear una mejor vida y un mundo más armonioso. Dictadores como Adolfo Hitler y José Stalin pueden haber tenido mucho conocimiento $\mathrm{y}$ haber sido aun buenos pensado- 
res críticos, al menos con respecto a la perpetuación de su propio poder. Dada la definición de sabiduría, sin embargo, sería difícil argumentar que eran sabios.

d) Finalmente, los estudiantes -quienes luego se convertirán en padres y líderes-son siempre parte de una gran comunidad, y por lo tanto se beneficiarán de aprender a juzgar correctamente, con validez o con justicia en nombre de su comunidad.

Es muy posible que el futuro continuará asediado por conflictos, y que la mayoría de los estudiantes de la actualidad en algún momento u otro de sus vidas se encontrarán con dilemas difíciles. Por ello, considero que no solo se debe enseñar a los estudiantes a recordar hechos y pensar críticamente (y aun creativamente) acerca de sus vidas y el contenido de las materias que aprenden en la escuela, sino que deben pensar sabiamente acerca de ello también.

\section{Referencias}

Baltes, P. B., \& Staudinger, U. (1993). The search for a psychology of wisdom. Current Directions in Psychological Science, 2, 75-80.

Baltes, P. B., \& Staudinger, U. (2000). Wisdom: A metaheuristic (pragmatic) to orchestrate mind and virtue toward excellence. American Psychologist, 55, 122-136.

Birren, J. E., \& Fisher, L. M. (1990). The elements of wisdom: Overview and integration. En R. J. Sternberg (Ed.), Wisdom: Its nature, origins, and development (pp. 317-332). Nueva York: Cambridge University Press.

Clayton, V. (1975). Erickson's theory of human development as it applies to the aged: Wisdom as contradictory cognition. Human Development, 18, 119-128.

Clayton, V. (1982). Wisdom and intelligence: The nature and function of knowledge in the later years. International Journal of Aging and Development, 15, 315-321.

Csikszentmihalyi, M., \& Rathunde, K. (1990). The psychology of wisdom: An evolutionary interpretation. En R. J. Sternberg (Ed.), Wisdom: Its nature, origins, and development (pp. 25-51). Nueva York: Cambridge University Press.

Dweck, C. S., \& Leggett, E. L. (1988). A social-cognitive approach to motivation and personality. Psychological Review, 95, 256-273.

Flavell, J. H. (1987). Speculations about the nature and development of metacognition. En F. E. Wienert \& R. H. Kluwe (Eds.), Metacognition, motivation, and understanding (pp. 21-29). Hillsdale, NJ: Erlbaum.

Holliday, S. G., \& Chandler, M. J. (1986). Wisdom: Explorations in adult competence. Basilea, Suiza: Karger. 
Kitchener, K. S., \& Brenner, H. G. (1990). Wisdom and reflective judgment: Knowing in the face of uncertainty. En R. J. Sternberg (Ed.), Wisdom: Its Nature, origins, and development (pp. 212-229). Nueva York: Cambridge University Press.

Kramer, D. A. (1990). Conceptualizing wisdom: The primacy of affect-cognition relations. En R. J. Sternberg (Ed.), Wisdom: Its nature, origins, and development (pp. 279-313). Nueva York: Cambridge University Press.

Kuhn, D., Shaw, V., \& Felton, M. (1997). Effects of dyadic interaction on argumentative reasoning. Cognition and Instruction, 15, 287-315.

Labouvie-Vief, G. (1990). Wisdom as integrated thought: Historical and developmental perspectives. En R. J. Sternberg (Ed.), Wisdom: Its Nature, origins, and development (pp. 52-83). Nueva York: Cambridge University Press.

McDevitt, T. M. (1990). Mothers' and children's beliefs about listening. Child Study Journal, 20, 105-128.

Paris, S. G. (2001). Wisdom, snake oil, and the educational marketplace. Educational Psychologist, 36, 257260.

Pascual-Leone, J. (1990). An essay on wisdom: Toward organismic processes that make it possible. En R.
J. Sternberg (Ed.), Wisdom: Its nature, origins, and development (pp. 244-278). Nueva York: Cambridge University Press.

Perkins, D. (2002). The engine of folly. En R. J. Sternberg (Ed.), Why smart people can be so stupid (pp. 233243). New Haven, CT: Yale University Press.

Piaget, J. (1972). The psychology of intelligence. Totowa, NJ: LittlefieldAdams.

Polanyi, M. (1966). The tacit dimensions. Garden City, NY: Doubleday.

Qian, G., \& Alvermann, D. E. (2000). Relationship between epistemological beliefs and conceptual change learning. Reading \& Writing Quarterly, 16, 59-74.

Reznitskaya, A., Anderson, R. C., McNurlen, B., Nguyen-Jahiel, K., Archodidou, A., \& Kim, S. (2001). Influence of oral discussion on written argument. Discourse Processes, $32,155-175$.

Reznitskaya, A., \& Sternberg, R. J. (2004). Teaching students to make wise judgments: The "teaching for wisdom” program. En P. A. Linley, \& S. Joseph (Eds.), Positive psychology in practice, (pp. 181-196). Nueva York: Wiley.

Robinson, D. N. (1990). Wisdom through the ages. En R. J. Sternberg (Ed.), Wisdom: Its nature, ori- 
gins, and development. Nueva York: Cambridge University Press.

Smith, J. \& Baltes, P. B. (1990). Wisdom-related knowledge: Age/cohort differences in response to life-planning problems. Developmental Psychology, 26, 494-505.

Songer, N. B., \& Linn, M. C. (1991). How do views of science influence knowledge integration. Journal of Research in Science Teaching, 28, 761-784.

Sternberg, R. J. (1990). Wisdom: Its nature, origins, and development. Nueva York: Cambridge University Press.

Sternberg, R. J. (1990). Understanding wisdom. En R. J. Sternberg (Ed.), Wisdom: Its nature, origins, and development. Nueva York: Cambridge University Press.

Sternberg, R. J. (1998). A balance theory of wisdom. Review of General Psychology, 2, 347-365.

Sternberg, R. J. (2001). Why schools should teach for wisdom: The balance theory of wisdom in educational settings. Educational Psychologist, $36,227-245$.
Sternberg, R. J. (2003). Rediscovering wisdom. Psychology Teacher Network, 13(1), 1-2.

Sternberg, R. J. (2004). Teaching for wisdom: What matters is not what students know, but how they use it. En D. R. Walling (Ed.), Public education, democracy, and the common good (pp. 121-132). Bloomington, IN: Phi Delta Kappan.

Sternberg, R. J., Forsythe, G. B., Hedlund, J., Horvath, J. A., Wagner, R. K., \& Williams, W. M., et al. (2000). Practical intelligence in everyday life. Cambridge, MA: Cambridge University Press.

Sternberg, R. J., \& Grigorenko, E. L. (2007). Teaching for successful intelligence (2. ${ }^{\mathrm{a}}$ ed.). Thousand Oaks, CA: Corwin.

Sternberg, R. J. \& Jordan, J. (2005) Handbook of wisdom: Psychological perspectives. Nueva York: Cambridge University Press.

Sternberg, R. J., \& Stemler, S. E. (2004). Wisdom as a moral virtue. En T. A. Thorkildsen, \& H. J. Walberg (Eds.), Nurturing morality (pp. 187-197). Nueva York: Kluwer Academic/Plenum Publishers. 